\title{
Long non-coding RNA expression profile in cervical cancer tissues
}

\author{
HUA ZHU $^{1,2}$, XIANGJIAN CHEN ${ }^{3}$, YAN HU ${ }^{2}$, ZHENGZHENG SHI $^{2}$, \\ QING ZHOU ${ }^{2}$, JINGJIE ZHENG ${ }^{2}$ and YIFENG WANG ${ }^{1}$ \\ ${ }^{1}$ Department of Obstetrics and Gynecology, Zhujiang Hospital, Southern Medical University, Guangzhou, Guangdong 510280; \\ Departments of ${ }^{2}$ Obstetrics and Gynecology, and ${ }^{3}$ Endoscopic Surgery, The First Affiliated Hospital \\ of Wenzhou Medical University, Wenzhou, Zhejiang 325000, P.R. China
}

Received September 14, 2015; Accepted March 23, 2017

DOI: $10.3892 / \mathrm{ol} .2017 .6319$

\begin{abstract}
Cervical cancer (CC), one of the most common types of cancer of the female population, presents an enormous challenge in diagnosis and treatment. Long non-coding (lnc)RNAs, non-coding (nc)RNAs with length $>200$ nucleotides, have been identified to be associated with multiple types of cancer, including CC. This class of nc transcripts serves an important role in tumor suppression and oncogenic signaling pathways. In the present study, the microarray method was used to obtain the expression profile of lncRNAs and protein-coding mRNAs and to compare the expression of lncRNAs between CC tissues and corresponding adjacent non-cancerous tissues in order to screen potential lncRNAs for associations with CC. Overall, 3356 lncRNAs with significantly different expression pattern in $\mathrm{CC}$ tissues compared with adjacent non-cancerous tissues were identified, while 1,857 of them were upregulated. These differentially expressed lncRNAs were additionally classified into 5 subgroups. Reverse transcription quantitative polymerase chain reactions were performed to validate the expression pattern of 5 random selected lncRNAs, and 2 lncRNAs were identified to have significantly different expression in CC samples compared with adjacent non-cancerous tissues. This finding suggests that those IncRNAs with different expression may serve important roles in the development of $\mathrm{CC}$, and the expression data may provide information for additional study on the involvement of lncRNAs in CC.
\end{abstract}

\section{Introduction}

Cervical cancer (CC) is the second major cause of female cancer-associated mortalities worldwide, which accounts for $\sim 12 \%$ of all female mortalities due to cancer $(1,2)$. A total of $\sim 49,000$ incident cases of CC were diagnosed in 2011, which

Correspondence to: Dr Yifeng Wang, Department of Obstetrics and Gynecology, Zhujiang Hospital, Southern Medical University, Guangzhou, Guangdong 510280, P.R. China

E-mail:wangyifeng2015@sohu.com

Key words: long non-coding RNA, expression profiles, cervical cancer occurred primarily in developing countries (3). At present, surgery, radiotherapy and chemotherapy remain the standard treatment for patients with CC. Clinical outcomes vary greatly between patients and are difficult to predict $(4,5)$. In addition, the clinical stage of disease is important regarding the prognosis for patients with $\mathrm{CC}$, and the 5-year survival rate for all stages combined is $\sim 70 \%$ (6). Therefore, it is urgently required to identify novel and effective biomarkers for early stage diagnosis and for potential targets for CC.

The human genome contains $>20,000$ protein-coding genes according to high-throughput transcriptome analysis, which represents $\sim 2 \%$ of the whole genome $(7,8)$. In addition, the rest of human genome may be transcribed into various short or long non-coding RNAs (lncRNAs) (9). IncRNAs are a subgroup of RNAs that are $>200$ nucleotides in length and may be implicated in various types of gene regulation, including transcriptional, post-transcriptional or epigenetic regulation (10). Additionally, these types of regulation implicated by lncRNAs may induce the progression of cancer or other diseases (11). Compared with short non-coding RNAs, like miRNAs, the biological roles of lncRNAs have largely been underestimated. However, there have already been studies characterizing the regulatory roles of lncRNAs, including cell proliferation, apoptosis and invasion, and parental imprinting (12-14). In addition, results from emerging studies have reported associations between the dysregulation of lncRNAs to multiple human diseases, including cancer (15-24). These studies have demonstrated that a large number of lncRNAs serve crucial roles in the progression of colorectal, breast, prostate and liver cancer, and other human tumors (25-28).

In the present study, the lncRNAs and mRNA expression profiles in $\mathrm{CC}$ tissues were compared with adjacent non-cancerous tissues. The differently expressed lncRNAs were additionally studied with potential gene targets through gene ontology (GO) and pathway analysis, and these results were confirmed in $40 \mathrm{CC}$ and adjacent non-cancerous tissues using reverse transcription quantitative polymerase chain reaction (RT-qPCR).

\section{Materials and methods}

Ethics statement. The present study was approved for the use of human biopsy samples by the Institution Review Board of Wenzhou Medical University (Wenzhou, China). The written 
consent was received from all participants in the present study at the time of surgery.

Clinical samples. A total of one CC tissue and one adjacent non-cancerous tissue from 3 patients were included in the microarray assay. Tissue samples from 43 patients (age range 20-65 years; average age 47.8 years) with cervical cancer between March 2011 and December 2013 were collected (Table I). All tissue samples were collected during surgical resection at the First Affiliated Hospital of Wenzhou Medical University and stored at $-80^{\circ} \mathrm{C}$ in the tissue bank for further use.

RNA extraction. TRIzol (Invitrogen; Thermo Fisher Scientific, Inc., Waltham, MA, USA) was used to extract the total RNAs from tissues. The mirVana miRNA Isolation kit (Ambion; Thermo Fisher Scientific, Inc.) was used to purify small RNAs in accordance with the manufacture's protocol. The concentration and purity of RNAs were determined by OD260/280 readings using a spectrophotometer (NanoDrop ND-2000; NanoDrop Technologies; Thermo Fisher Scientific, Inc., Pittsburgh, PA, USA). By using the RNA 6000 Nano Lab-on-a-Chip kit and the Bioanalyzer 2100 (Agilent Technologies, Inc., Santa Clara, CA, USA), RNA integrity was determined by capillary electrophoresis.

DNA microarray. Microarray assays were performed by Kangcheng Biotechnology Co. (Shanghai, China). Arraystar human IncRNA and mRNA Array version 3.0 (Qiagen $\mathrm{GmbH}$, Hilden, Germany) was used in the assay, which was designed with four identical arrays per slide $(4 \times 180 \mathrm{~K}$ format). Arraystar human lncRNA and mRNA Array contains 30,586 human lncRNAs probes and 26,109 human mRNAs probes, which were collected from a number of sources, including GENCODE/ENSEMBL (http://www.gencodegenes. org/data_ensembl.html), the Human lncRNA Catalog (17), RefSeq (https://www.ncbi.nlm.nih.gov/refseq/), USCS Genome Browser (http://genome.ucsc.edu/cgi-bin/hgGateway), Non-coding (nc) RNA Expression Database (http://nred. matticklab.com/cgi-bin/ncrnadb.pl), Antisense ncRNA pipeline (http://research.imb.uq.edu.au/rnadb/rnadb2_archive. $\mathrm{htm}$ ), homeotic gene ncRNAs and the ultra-conserved regions. Each RNA was detected by corresponding probes in duplicate experiments.

RNA amplification, labeling and hybridization. By using Eberwine's linear RNA amplification method and subsequent enzymatic reaction (29), fluorescent dye (Cy5 and Cy3) labeled complimentary (c)DNAs were produced using the CapitalBio cRNA Amplification and Labeling kit (CapitalBio Corporation, Beijing, China), according to the manufacturer's instructions.

Microarray imaging and data analysis. GeneSpring software (version 12.0; Agilent Technologies, Inc.) was applied to analyze the lncRNAs and mRNA array data. Threshold values of $\geq 2$ and $\leq$-2-fold change were used to identify the differentially expressed genes. The data was $\log (2)$ transformed and median centered by genes using the Adjust Data function of Multiexperiment Viewer software (MeV 4.3.02) (Dana-Farber Cancer Institute, Boston, MA, USA). The data
Table I. Clinicopathological characteristics in 43 patient samples of cervical cancer.

\begin{tabular}{lc} 
Clinical parameter & Number of cases \\
\hline Age (years) & \\
$<30$ & $3(7.0)$ \\
$30-55$ & $29(67.4)$ \\
$>55$ & $11(25.6)$ \\
Age at first birth (years) & \\
$<18$ & $2(4.7)$ \\
$18-24$ & $10(23.2)$ \\
$>24$ & $23(53.5)$ \\
Nulliparous & $8(18.6)$ \\
Caesarean section & \\
Never & $34(79.1)$ \\
Ever & $9(20.9)$ \\
Tumor types & \\
Endophytic type & $6(14.0)$ \\
Ulcerative type & $9(20.9)$ \\
Endocervical type & $11(25.6)$ \\
Exophytic type & $17(39.5)$
\end{tabular}

were subsequently analyzed with hierarchical clustering with average linkage. GO and pathway analysis were performed on Gene-Cloud of Biotechnology Information (GCBI) (https://www.gcbi.com.cn/gclib/html/index) according the protocol of the manufacturer.

$R T$-qPCR analysis. Total RNA of 40 clinical samples was isolated using TRIzol reagent (Invitrogen; Thermo Fisher Scientific, Inc.). The purity and concentration of RNA samples were determined by a spectrophotometer (NanoDrop ND-2000; Thermo Fisher Scientific, Inc.). The samples with an optical density 260/280 ratio $>1.8$ were reversely transcribed using a GoScript ${ }^{\mathrm{TM}}$ Reverse Transcription system kit (Promega Corporation, Madison, WI, USA) according to the manufacturer's protocol. The expression of selected lncRNAs were analyzed using qPCR with a GoTaq ${ }^{\circledR}$ qPCR Master Mix kit (Promega Corporation) on the StepOne Plus PCR system (Applied Biosystems; Thermo Fisher Scientific, Inc.). PCR reactions were performed using standard cycling parameters (stage 1: $50^{\circ} \mathrm{C}$ for $2 \mathrm{~min}$, Stage $2: 95^{\circ} \mathrm{C}$ for $10 \mathrm{~min}$ then 40 cycles of $95^{\circ} \mathrm{C}$ for $15 \mathrm{Sec}$ and $60^{\circ} \mathrm{C}$ for $1 \mathrm{~min}$ ). $\beta$-actin was used as an internal control for normalization, and the $2^{-\Delta \Delta \mathrm{Ct}}$ method (30) was used to calculate the expression of IncRNAs. Each reaction was performed 3 times. The sequences of the primers for qPCR are listed in Table II.

Statistical analysis. All data are expressed as the mean \pm standard deviation. $\mathrm{P}<0.05$ was considered to indicate a statistically significant difference. Comparison between groups was analyzed by a Student's t-test. All the statistical analyses were conducted using GraphPad Prism (version 5; GraphPad Software, Inc., La Jolla, CA, USA). 
Table II. Primers for quantitative reverse transcription polymerase chain reaction.

Sequence name (ID)

$\beta$-actin

TCONS_00027301

(hsaLB_LI_107288)

TCONS_00029064

(hsaLB_LI_114731)

TCONS_00010587

(hsaLB_LI_38766)

TCONS_00003380

(hsaLB_LI_16629)

TCONS_00026907

(hsaLB_LI_16629)

Forward primer (5'-3')

Reverse primer (5'-3')

ATCGTGCGTGACATTAAGGAGAAG
GCCGACAAAGAGAAGGGAAGA

TGCTGCCGGAAACGTGTG

CACCACCAAGACCCCCTCAC

GCGAACAAGCGTCCAGGTAA

TGGATTGTTGGGTATATTTTGGA
AGGAAGGAAGGCTGGAAGAGTG

GCAGATAGTGAAGGCATGGAAGT

GGCTTCTGGGGAGAGTGGG

GCGAACAAGCGTCCAGGTAA

CGGCACAGAAGGAATCCAAC

TGTATGAAGAGGATGCTGAAGGC

Lnc, long non-coding. In the parentheses are the lncRNA accession IDs for lncRNAs from the lncRBase (http://bicresources.jcbose. ac.in/zhumur/lncrbase/).

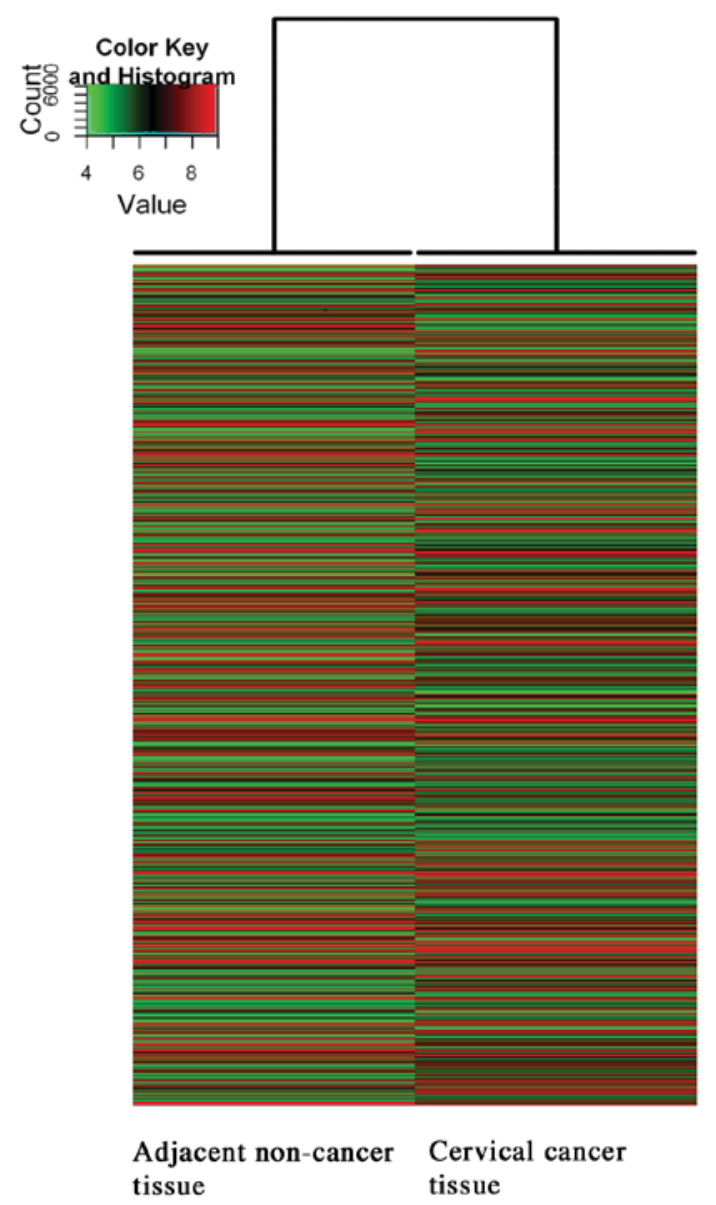

Figure 1. Hierarchical clustering analysis of differentially expressed long non-coding RNAs and mRNAs between cervical cancer tissues and adjacent non-cancerous tissues. Red color indicates an upregulation of transcripts and green color indicates transcripts that are downregulated.

\section{Results}

Overview of the IncRNA and mRNA profiles in CC tissues and adjacent non-cancerous tissues. In the present study, a commercial human IncRNA microarray (Kangcheng Biotechnology Co.) was used to investigate the characteristic expression profiles of $\mathrm{CC}$ tissues and adjacent non-cancerous tissues by using total RNA isolated from different patients with $\mathrm{CC}$. The lncRNA profiles of CC tissues were analyzed by comparing $\mathrm{CC}$ tissues with adjacent non-cancerous tissues.

Hierarchical clustering analysis was used to investigate the expression level of the lncRNAs (Fig. 1 and Table III). The results of the IncRNA expression profiles are summarized in Table III. The alternation of lncRNA expressions were evaluated by fold change. As demonstrated in Table III, 32,829 lncRNAs on the microarray exhibited expression above background levels, and $10.22 \%(3,356 / 32,829)$ of the lncRNAs were significantly differentially expressed between the CC sample and corresponding adjacent non-cancerous sample (absolute fold-change $\geq 2)$. A total of $55.33 \%(1,857 / 3,356)$ of the significantly differentially expressed lncRNAs were upregulated in the CC tissue. By contrast, $8.25 \%(1,987 / 24,063)$ of the mRNAs demonstrated significantly different expression between cancer and corresponding adjacent non-cancerous tissues (absolute fold-change $\geq 2$ ), whilst 56.72\% $(1,127 / 1,987$ ) of the mRNAs were significantly upregulated.

Analysis of the distribution of differentially expressed lncRNAs and protein-coding mRNAs reveals that the lncRNAs and mRNAs were not distributed equally on each chromosome (Fig. 2). The analysis reveals that chromosome 1 had the highest number of differentially expressed altered lncRNAs and protein-coding mRNAs (Fig. 2). All differentially expressed protein-coding genes were submitted for GO term enrichment analysis. A total of three domains were studied, including biological process, cellular component and molecular function (Fig. 3).

Classification and subgroup analysis of lncRNAs. IncRNAs may be classified into five subgroups, including sense lncRNAs, antisense lncRNAs, intronic lncRNAs, intergenic lncRNAs and bidirectional lncRNAs based on different transcription forms. Sense lncRNAs exhibit the same transcriptional direction with exons of protein-coding genes, and 
Table III. Number of differentially expressed lncRNAs and mRNAs in cervical cancer and corresponding adjacent non-cancerous tissues.

\begin{tabular}{|c|c|c|c|c|}
\hline Parameter & $\mathrm{n}(\%)$ & Type of change & Fold change & $\mathrm{n}(\%)$ \\
\hline Total lncRNAs on the microarray & 32,829 & & & \\
\hline Differentially expressed lncRNAs & $3,356(10.22)$ & $\begin{array}{l}\text { Up } \\
\text { Down }\end{array}$ & $\begin{array}{l}\geq 2 \\
\geq 2\end{array}$ & $\begin{array}{l}1,857(55.33) \\
1,499(44.67)\end{array}$ \\
\hline Total mRNAs & 24,063 & & & \\
\hline Differentially expressed mRNAs & $1,987(8.25)$ & $\begin{array}{l}\text { Up } \\
\text { Down }\end{array}$ & $\begin{array}{l}\geq 2 \\
\geq 2\end{array}$ & $\begin{array}{r}1,127(56.72) \\
860(43.28)\end{array}$ \\
\hline
\end{tabular}

lnc, long non-coding.

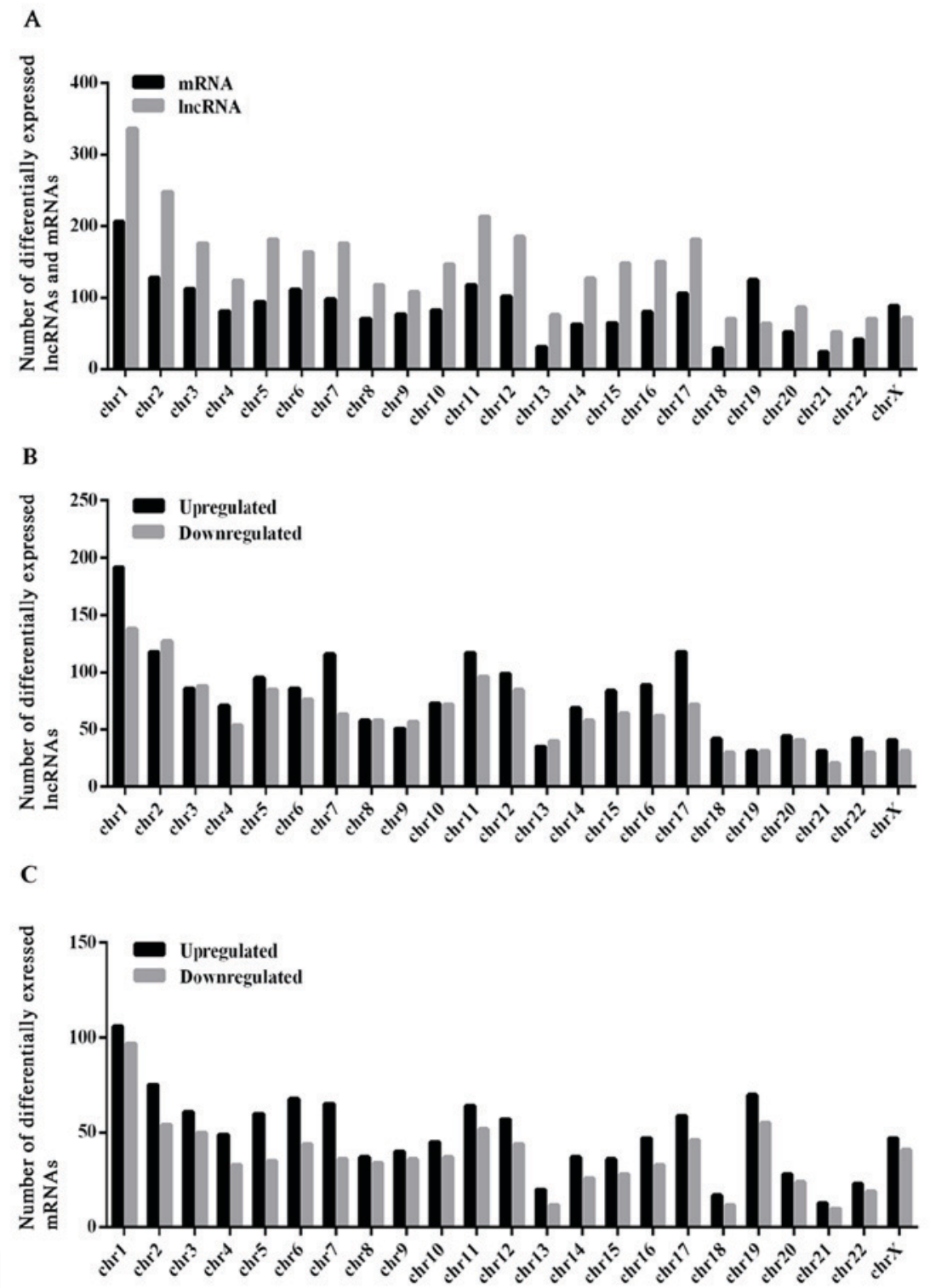

Figure 2. Distribution of differentially expressed lncRNAs and mRNAs on each chromosome. (A) The distribution of all differentially expressed lncRNAs and mRNAs on each chromosome. (B) The distribution of upregulated and downregulated lncRNAs on each chromosome. (C) The distribution of upregulated and downregulated mRNAs on each chromosome. lncRNA, long non-coding RNA; chr, chromosome.

a previous study identified that certain sense lncRNAs may be viewed as non-coding transcript variants of genes (31). These non-coding transcript variants may regulate gene expression (32). The present study identified 331 upregulated and 175 
A

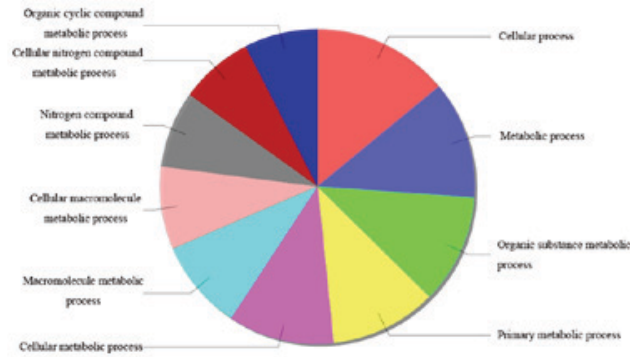

C

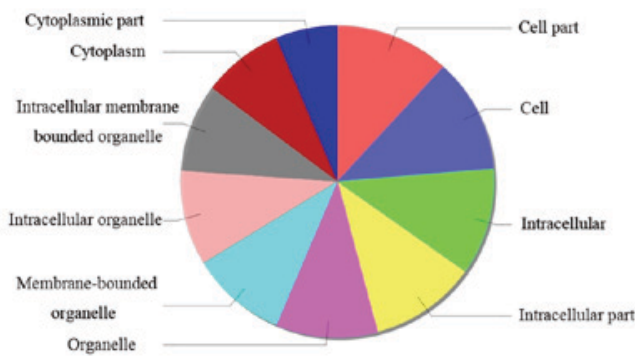

E GO Molecular Function Classification

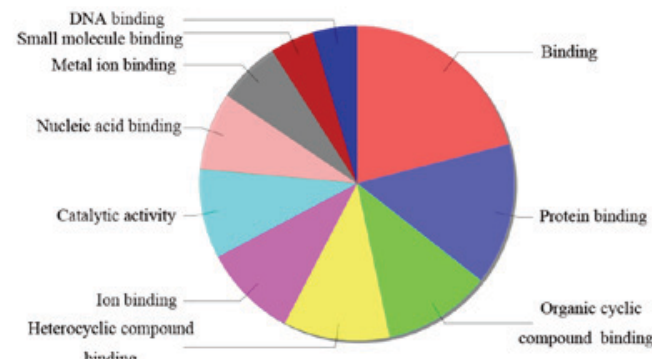

B GO Biological Process Classification

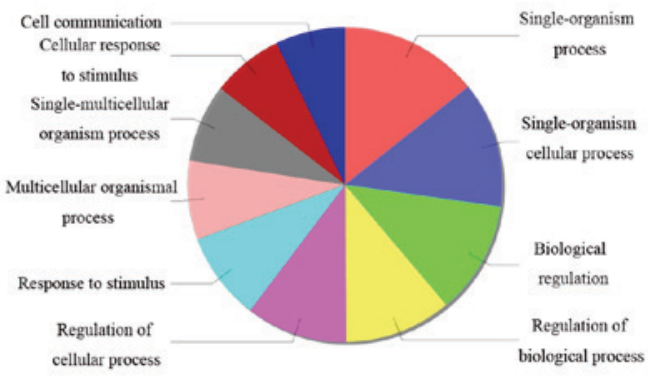

D GO Cellular Component Classification

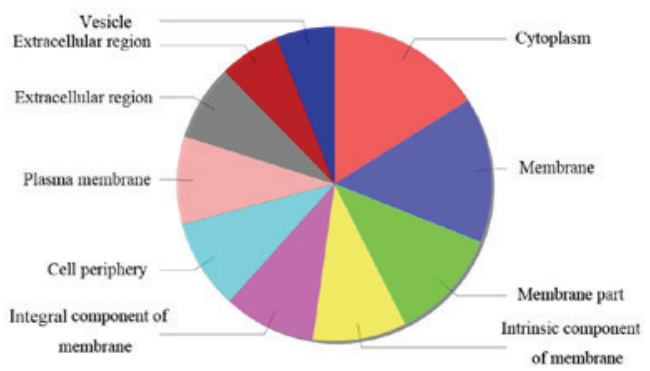

$\mathbf{F}$ GO Molecular Function Classification

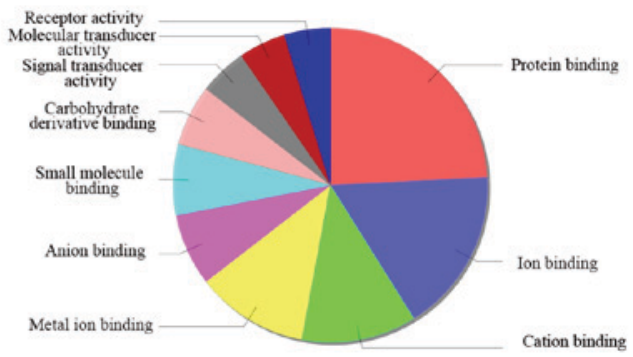

Figure 3. GO enrichment analysis of the differentially expressed mRNAs. Results for GO enrichment analysis for: (A) Biological process of upregulated mRNAs, (B) cellular component of upregulated mRNAs, (C) molecular function of upregulated mRNAs, (D) biological process of downregulated mRNAs, (E) cellular component of downregulated mRNAs and (F) molecular function of downregulated mRNAs. GO, gene ontology.

Table IV. Types of differentially expressed lncRNAs and mRNAs in cervical cancer tissues compared with corresponding adjacent non-cancerous samples.

\begin{tabular}{llcc}
\hline $\begin{array}{l}\text { Type of } \\
\text { lncRNAs }\end{array}$ & $\begin{array}{l}\text { Type of } \\
\text { change }\end{array}$ & $\begin{array}{c}\text { Fold } \\
\text { change }\end{array}$ & $\begin{array}{c}\text { Number of } \\
\text { lncRNAs }\end{array}$ \\
\hline Sense & Up & $\geq 2$ & 331 \\
Antisense & Down & $\geq 2$ & 175 \\
& Up & $\geq 2$ & 355 \\
Intronic & Down & $\geq 2$ & 263 \\
& Up & $\geq 2$ & 415 \\
Intergenic & Down & $\geq 2$ & 345 \\
\multirow{2}{*}{ Bidirectional } & Up & $\geq 2$ & 613 \\
& Down & $\geq 2$ & 808 \\
& Up & $\geq 2$ & 140 \\
& Down & $\geq 2$ & 63 \\
\hline
\end{tabular}

lnc, long non-coding. downregulated sense lncRNAs in CC tissues compared with adjacent non-cancerous tissues (Table IV).

There were 355 antisense IncRNAs significantly upregulated in CC tissues, and 263 antisense lncRNAs that were downregulated. Anti-sense lncRNAs are transcribed against overlapping genes, regulate their protein-coding counterparts via multiple mechanisms, including chromatin remodeling, alternative splicing, translational interference and promoter targeting (33).

Intronic lncRNAs have been demonstrated to regulate the expression of neighbourhood genes or other genes through alternative splicing, miRNA, RNA interference, transcriptional disrution and chromatin modification in previous studies $(34,35)$. The present study identified 415 upregulated and 345 downregulated intronic lncRNAs in CC tissues, as illustrated in Table IV.

Bidirectional lncRNAs may regulate the expression of their neighboring genes through epigenetic modification (36). Bidirectional non-coding genes share paired transcriptional initiation sites with separate transcripts, which are in 
A

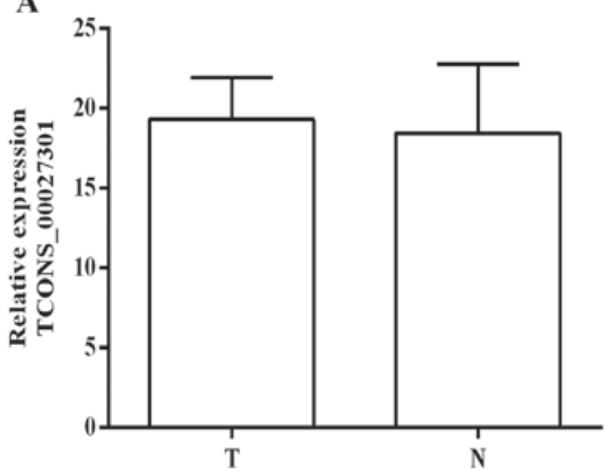

C

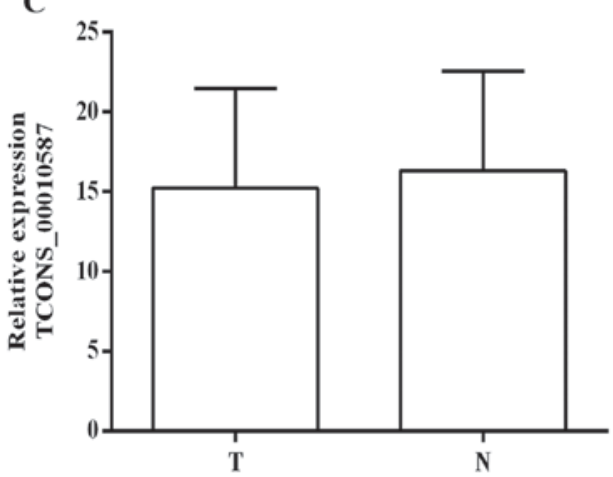

$\mathbf{E}$

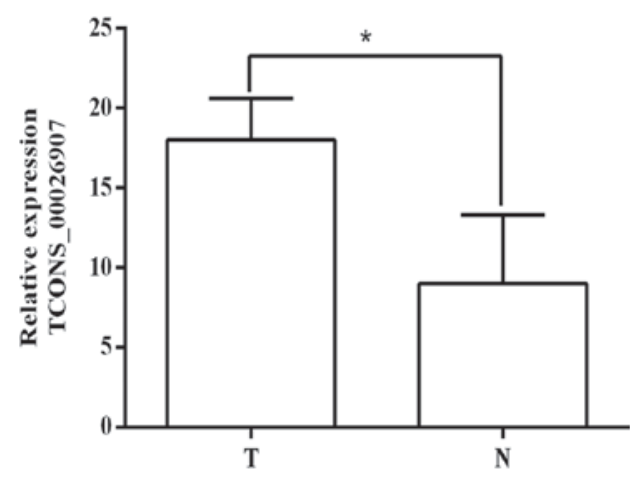

B

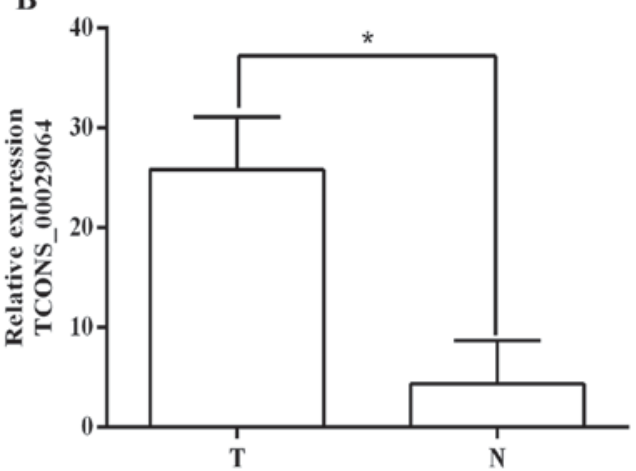

D

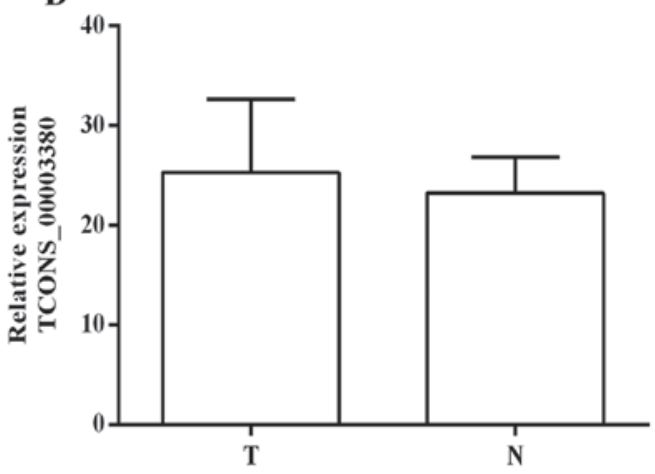
merase chain reaction. (A) TCONS_00027301. (B) TCONS_00029064. (C) TCONS_00010587. (D) TCONS_0,0003380. (E) TCONS_00026907. "P<0.05; N non-cancerous cervical tissues; $\mathrm{T}$, cervical cancer samples.

opposite orientations, but with close proximity (37). Among the significantly changed lncRNAs in the present study, 140 bidirectional lncRNAs were upregulated and 63 were downregulated.

Intergenic non-coding RNAs are able to regulate the expression of target genes with a distance $>10 \mathrm{~kb}$ through recruiting histone-modifying enzymes to the chromatin. Therefore, the target genes of intergenic non-coding RNAs may be located across the genome. The present study identified 613 upregulated and 808 downregulated intergenic lncRNAs in CC tissues compared with adjacent non-cancerous tissues.

Validation of the significantly changed lncRNAs by RT-qPCR. The expression level of differentially expressed lncRNAs was confirmed using RT-qPCR, (Fig. 4) and a total of 5 lncRNAs that were particularly markedly upregulated were selected. The result demonstrates that 3 of the selected lncRNAs did not exhibit an altered expression pattern. By contrast, 2 lncRNAs
(TCONS_00029064 and TCONS_00026907) were significantly upregulated in CC tissues compared with adjacent non-cancerous tissues (Fig. 4), which was consistent with the microarray result (Fig. 1). RT-qPCR analysis indicates that TCONS_00029064 and TCONS_00026907 may serve important roles in $\mathrm{CC}$ tumorigenesis and other associated biological process.

\section{Discussion}

CC remains an enormous challenge and worldwide public health problem (38). Despite the development of advanced therapeutic strategies, the prognosis in patients with $\mathrm{CC}$ varies and is difficult to predict. Therefore, novel molecular mechanisms are needed in order to develop effective therapeutic strategies. In previous studies protein-coding genes and non-coding RNAs have been reported to be involved in the molecular mechanism of carcinogenesis $(39,40)$. Compared to 
the knowledge of coding genes and short non-coding RNAs, such as miRNAs, general understanding of lncRNAs remains limited. An increasing number of studies demonstrate that certain lncRNAs serve crucial roles in cancer and associated biological functions, including cell migration/invasion (41) and cell-cycle regulation (42). Non-coding RNAs, including SPRY4-IT1, have been reported to have a key role in cell growth and differentiation in melanoma cell lines (43). Additionally, the altered expression of a number of non-coding RNAs have been associated with cancer progression (44). Despite the previous findings, the associations between lncRNA and CC remain unknown.

In the present study, the overall lncRNAs expression profile of $\mathrm{CC}$ tissues was established by comparing the expression of lncRNAs in CC samples with the expression in corresponding adjacent non-cancerous tissues. A total of 3,356 differentially expressed lncRNAs, and a total of 1,987 differentially expressed mRNAs were revealed. Based on the association of the lncRNAs with coding genes, the lncRNAs may be classified into five subgroups that include sense, antisense, intronic, bidirectional and intergenic lncRNAs (45). The results of the present study indicated that the different subtypes of differentially expressed IncRNAs are unequally distributed across the genome, which is consistent with a previous study (46). Although all the five subgroups of lncRNAs were detected, intergenic lncRNAs constituted a major portion. This result indicates that among differentially expressed lncRNAs, intergenic lncRNAs are more abundant compared with others, which suggests that intergenic lncRNAs may serve important roles in CC.

Overall, the present study characterized the expression profile of lncRNAs and mRNAs in CC tissues and corresponding adjacent non-cancerous tissues using microarray and identified a large portion of differentially expressed lncRNAs and mRNAs. These results remain limited due to sample quantities. Further studies using greater numbers of $\mathrm{CC}$ samples may provide more accurate expression information for lncRNAs and mRNAs. Meanwhile, the data of the present study will facilitate other groups to better understand the function of lncRNAs in CC development and metastasis.

\section{Acknowledgements}

The present study was supported by grants from the Medical and Health Project of Zhejiang Province (grant no. 2016KYA137 to Dr H.Z), Wenzhou Public Welfare Science and Technology Project (grant no. Y20140707 to Dr H.Z) and the Incubation Project of the First Affiliated Hospital of Wenzhou Medical University (grant no. FHY2014009 to Dr H.Z).

\section{References}

1. Jemal A, Bray F, Center MM, Ferlay J, Ward E and Forman D: Global cancer statistics. CA Cancer J Clin 61: 69-90, 2011.

2. Ellenson LH and Wu TC: Focus on endometrial and cervical cancer. Cancer Cell 5: 533-538, 2004.

3. Schiffman M, Wentzensen N, Wacholder S, Kinney W, Gage JC and Castle PE: Human papillomavirus testing in the prevention of cervical cancer. J Natl Cancer Inst 103: 368-383, 2011.

4. Waggoner SE: Cervical cancer. Lancet 361: 2217-2225, 2003.

5. Biewenga P, van der Velden J, Mol BW, Stalpers LJ, Schilthuis MS, van der Steeg JW, Burger MP and Buist MR: Prognostic model for survival in patients with early stage cervical cancer. Cancer 117: 768-776, 2011
6. Shingleton HM, Jones WB, Russell A, Fremgen A, Chmiel JS, Ocwieja K, Winchester DP and Clive R: Hysterectomy in invasive cervical cancer: A national patterns of care study of the American College of Surgeons. J Am Coll Surg 183: 393-400, 1996.

7. Nagano $T$ and Fraser P: No-nonsense functions for long noncoding RNAs. Cell 145: 178-181, 2011.

8. Guttman M, Amit I, Garber M, French C, Lin MF, Feldser D, Huarte M, Zuk O, Carey BW, Cassady JP, et al: Chromatin signature reveals over a thousand highly conserved large non-coding RNAs in mammals. Nature 458: 223-227, 2009.

9. ENCODE Project Consortium; Birney E, Stamatoyannopoulos JA, Dutta A, Guigó R, Gingeras TR, Margulies EH, Weng Z, Snyder M, Dermitzakis ET, et al: Identification and analysis of functional elements in $1 \%$ of the human genome by the ENCODE pilot project. Nature 447: 799-816, 2007.

10. Tsai MC, Spitale RC and Chang HY: Long intergenic noncoding RNAs: New links in cancer progression. Cancer Res 71: 3-7, 2011.

11. Maruyama R, Shipitsin M, Choudhury S, Wu Z, Protopopov A, Yao J, Lo PK, Bessarabova M, Ishkin A, Nikolsky Y, et al: Altered antisense-to-sense transcript ratios in breast cancer. Proc Natl Acad Sci USA 109: 2820-2824, 2012.

12. Loewer S, Cabili MN, Guttman M, Loh YH, Thomas K, Park IH, Garber M, Curran M, Onder T, Agarwal S, et al: Large intergenic non-coding RNA-RoR modulates reprogramming of human induced pluripotent stem cells. Nat Genet 42: 1113-1117, 2010.

13. Mercer TR, Dinger ME and Mattick JS: Long non-coding RNAs: Insights into functions. Nat Rev Genet 10: 155-159, 2009.

14. Villegas VE, Rahman MF, Fernandez-Barrena MG, Diao Y, Liapi E, Sonkoly E, Ståhle M, Pivarcsi A, Annaratone L, Sapino A, et al: Identification of novel non-coding RNA-based negative feedback regulating the expression of the oncogenic transcription factor GLI1. Mol Oncol 8: 912-926, 2014.

15. Liu XH, Liu ZL, Sun M, Liu J, Wang ZX and De W: The long non-coding RNA HOTAIR indicates a poor prognosis and promotes metastasis in non-small cell lung cancer. BMC Cancer 13: 464, 2013.

16. Lu X, Fang Y, Wang Z, Xie J, Zhan Q, Deng X, Chen H, Jin J, Peng C, Li H and Shen B: Downregulation of gas5 increases pancreatic cancer cell proliferation by regulating CDK6. Cell Tissue Res 354: 891-896, 2013.

17. Kong GY, Zhang JP, Zhang S, Shan CL, Ye LH and Zhang XD: Hepatitis B virus $X$ protein promotes hepatoma cell proliferation via upregulation of MEKK2. Acta Pharmacol Sin 32: 1173-1180, 2011.

18. Yuan SX, Yang F, Yang Y, Tao QF, Zhang J, Huang G, Yang Y, Wang RY, Yang S, Huo XS, et al: Long noncoding RNA associated with microvascular invasion in hepatocellular carcinoma promotes angiogenesis and serves as a predictor for hepatocellular carcinoma patients' poor recurrence-free survival after hepatectomy. Hepatology 56: 2231-2241, 2012.

19. Lu KH, Li W, Liu XH, Sun M, Zhang ML, Wu WQ, Xie WP and Hou YY: Long non-coding RNA MEG3 inhibits NSCLC cells proliferation and induces apoptosis by affecting p53 expression. BMC Cancer 13: 461, 2013.

20. Huarte M, Guttman M, Feldser D, Garber M, Koziol MJ, Kenzelmann-Broz D, Khalil AM, Zuk O, Amit I, Rabani M et al: A large intergenic noncoding RNA induced by p53 mediates global gene repression in the p53 response. Cell 142: 409-419, 2010.

21. Schmidt LH, Spieker T, Koschmieder S, Schäffers S, Humberg J, Jungen D, Bulk E, Hascher A, Wittmer D, Marra A, et al: The long noncoding MALAT-1 RNA indicates a poor prognosis in non-small cell lung cancer and induces migration and tumor growth. J Thorac Oncol 6: 1984-1992, 2011.

22. Matouk IJ, DeGroot N, Mezan S, Ayesh S, Abu-lail R, Hochberg A and Galun E: The H19 non-coding RNA is essential for human tumor growth. PLoS One 2: e845, 2007.

23. Wang XS, Zhang Z, Wang HC, Cai JL, Xu QW, Li MQ, Chen YC, Qian XP, Lu TJ, Yu LZ, et al: Rapid identification of UCA1 as a very sensitive and specific unique marker for human bladder carcinoma. Clin Cancer Res 12: 4851-4858, 2006.

24. de Kok JB, Verhaegh GW, Roelofs RW, Hessels D, Kiemeney LA, Aalders TW, Swinkels DW and Schalken JA: DD3(PCA3), a very sensitive and specific marker to detect prostate tumors. Cancer Res 62: 2695-2698, 2002.

25. Gao Y, Chen G, Zeng Y, Zeng J, Lin M, Liu X and Liu J: Invasion and metastasis-related long noncoding RNA expression profiles in hepatocellular carcinoma. Tumour Biol 36: 7409-7422, 2015. 
26. Ren S, Peng Z, Mao JH, Yu Y, Yin C, Gao X, Cui Z, Zhang J, Yi K, $\mathrm{Xu}$ W, et al: RNA-seq analysis of prostate cancer in the Chinese population identifies recurrent gene fusions, cancer-associated long noncoding RNAs and aberrant alternative splicings. Cell Res 22: 806-821, 2012.

27. Kogo R, Shimamura T, Mimori K, Kawahara K, Imoto S, Sudo T, Tanaka F, Shibata K, Suzuki A, Komune S, et al: Long noncoding RNA HOTAIR regulates polycomb-dependent chromatin modification and is associated with poor prognosis in colorectal cancers. Cancer Res 71: 6320-6326, 2011.

28. Yang Z, Zhou L, Wu LM, Lai MC, Xie HY, Zhang F and Zheng SS: Overexpression of long non-coding RNA HOTAIR predicts tumor recurrence in hepatocellular carcinoma patients following liver transplantation. Ann Surg Oncol 18: 1243-1250, 2011.

29. Phillips J and Eberwine JH: Antisense RNA Amplification: A Linear amplification method for analyzing the mRNA population from single living cells. Methods 10: 283-288, 1996.

30. Livak KJ and Schmittgen TD: Analysis of relative gene expression data using real-time quantitative PCR and the 2(-Delta Delta C(T)) method. Methods 25: 402-408, 2001.

31. Carninci P, Kasukawa T, Katayama S, Gough J, Frith MC, Maeda N, Oyama R, Ravasi T, Lenhard B, Wells C, et al: The transcriptional landscape of the mammalian genome. Science 309: 1559-1563, 2005.

32. Wang X, Arai S, Song X, Reichart D, Du K, Pascual G, Tempst P, Rosenfeld MG, Glass CK and Kurokawa R: Induced ncRNAs allosterically modify RNA-binding proteins in cis to inhibit transcription. Nature 454: 126-130, 2008.

33. Faghihi MA and Wahlestedt C: Regulatory roles of natural antisense transcripts. Nat Rev Mol Cell Biol 10: 637-643, 2009.

34. Louro R, El-Jundi T, Nakaya HI, Reis EM and Verjovski-Almeida S: Conserved tissue expression signatures of intronic noncoding RNAs transcribed from human and mouse loci. Genomics 92: 18-25, 2008.

35. Yang L, Lin C, Jin C, Yang JC, Tanasa B, Li W, Merkurjev D, Ohgi KA, Meng D, Zhang J, et al: IncRNA-dependent mechanisms of androgen-receptor-regulated gene activation programs. Nature 500: 598-602, 2013

36. Louro R, Smirnova AS and Verjovski-Almeida S: Long intronic noncoding RNA transcription: Expression noise or expression choice? Genomics 93: 291-298, 2009.
37. Trinklein ND, Aldred SF, Hartman SJ, Schroeder DI, Otillar RP and Myers RM: An abundance of bidirectional promoters in the human genome. Genome Res 14: 62-66, 2004.

38. Schiffman M, Castle PE, Jeronimo J, Rodriguez AC and Wacholder S: Human papillomavirus and cervical cancer. Lancet 370: 890-907, 2007.

39. Jin G, Sun J, Isaacs SD, Wiley KE, Kim ST, Chu LW, Zhang Z, Zhao H, Zheng SL, Isaacs WB and Xu J: Human polymorphisms at long non-coding RNAs (lncRNAs) and association with prostate cancer risk. Carcinogenesis 32: 1655-1659, 2011.

40. Nie W, Ge HJ, Yang XQ, Sun X, Huang H, Tao X, Chen WS and Li B: LncRNA-UCA1 exerts oncogenic functions in non-small cell lung cancer by targeting miR-193a-3p. Cancer Lett 371: 99-106, 2015

41. Gutschner T, Hämmerle M, Eissmann M, Hsu J, Kim Y, Hung G, Revenko A, Arun G, Stentrup M, Gross M, et al: The noncoding RNA MALAT1 is a critical regulator of the metastasis phenotype of lung cancer cells. Cancer Res 73: 1180-1189, 2013.

42. Kim T, Cui R, Jeon YJ, Fadda P, Alder H and Croce CM: MYC-repressed long noncoding RNÁs antagonize MYC-induced cell proliferation and cell cycle progression. Oncotarget 6: 18780-18789, 2015.

43. Khaitan D, Dinger ME, Mazar J, Crawford J, Smith MA, Mattick JS and Perera RJ: The melanoma-upregulated long noncoding RNA SPRY4-IT1 modulates apoptosis and invasion. Cancer Res 71: 3852-3862, 2011.

44. Lujambio A, Portela A, Liz J, Melo SA, Rossi S, Spizzo R, Croce CM, Calin GA and Esteller M: CpG island hypermethylation-associated silencing of non-coding RNAs transcribed from ultraconserved regions in human cancer. Oncogene 29: 6390-63401, 2010.

45. Ponting CP, Oliver PL and Reik W: Evolution and functions of long noncoding RNAs. Cell 136: 629-641, 2009.

46. Derrien T, Johnson R, Bussotti G, Tanzer A, Djebali S, Tilgner H, Guernec G, Martin D, Merkel A, Knowles DG, et al: The GENCODE v7 catalog of human long noncoding RNAs: Analysis of their gene structure, evolution, and expression. Genome Res 22: 1775-1789, 2012. 